\title{
Lattice misfit of high refractory ruthenium containing nickel-base superalloys
}

\author{
S. Neumeier ${ }^{1}$, J. Ang ${ }^{1}$, R.A. Hobbs ${ }^{2}$, C.M.F. Rae ${ }^{1}$ and H.J. Stone ${ }^{1}$ \\ ${ }^{1}$ Rolls-Royce University Technology Centre, Department of Materials Science \& Metallurgy, \\ University of Cambridge, Pembroke Street, Cambridge, CB2 3QZ, UK \\ ${ }^{2}$ Rolls-Royce plc., PO BOX 31, Derby, DE24 8BJ, UK \\ sn367@cam.ac.uk, hjs1002@cam.ac.uk
}

Keywords: Lattice misfit, X-ray diffraction, Ni-base superalloys, Ruthenium

\begin{abstract}
The influence of $\mathrm{Ru}, \mathrm{Co}$, Mo and $\mathrm{W}$ on the lattice misfit of eight highly alloyed Re containing single crystal nickel-base superalloys was investigated. High resolution X-ray diffraction (XRD) was used to relate the elemental partitioning behavior and the Vegard coefficients of the elements under investigation to the measured lattice parameter of the $\gamma$ and $\gamma^{\prime}$ phase. The residual chemical segregation and especially the coherency stress-induced tetragonal distortion of the $\gamma$ matrix lattice in the high Mo containing alloys results in the observation of two different lattice parameters for the $\gamma$ matrix phase. This leads to three overlapping, but clearly distinguishable $\{002\} X$-ray reflections.
\end{abstract}

\section{Introduction}

In order to meet the demand for more efficient gas turbines, single crystal nickel-base superalloys for turbine blade applications are increasingly alloyed with refractory elements like Re. However these highly alloyed nickel-base superalloys are prone to the formation of deleterious topologically close packed (TCP) phases. In the latest generation of single crystal nickel-base superalloys the platinum group metal $\mathrm{Ru}$ is also added as it enhances the phase stability of Ni-base superalloys. Accordingly, even higher levels of refractory elements may be added without the formation of TCP phases. This leads to an improvement of the temperature capability of advanced Ni-base superalloys [1].

As a direct consequence, these superalloys typically possess an increased lattice misfit, $\delta$, which is defined by the expression,

$$
\delta=\frac{2\left(a_{\gamma}{ }^{\left.\prime-a_{\gamma}\right)}\right.}{a_{\gamma}+a_{\gamma}}
$$

between the lattice parameters of the face centered, A1, matrix phase $(\gamma)$ and of the coherently embedded intermetallic, $\mathrm{L1}_{2}$, phase $\left(\gamma^{\prime}\right)$, compared to current alloys. As the lattice misfit induces coherency stresses, drives rafting of the $\gamma / \gamma^{\prime}$ microstructure at high temperatures and thereby influences the creep strength at the operating temperature $[1,2]$, knowledge of the lattice misfit is of great importance.

In this work the influence of $\mathrm{Ru}, \mathrm{Co}, \mathrm{W}$ and Mo on the lattice misfit of next generation nickel-base superalloys with high contents of Re has been investigated by means of high resolution X-ray diffraction. These four alloying elements were chosen since they are known to be important elements for next generation nickel-base superalloys. Ru, Co and especially $\mathrm{W}$ and Mo improve the creep strength and additionally both $\mathrm{Ru}$ and Co stabilize the $\gamma / \gamma^{\prime}$ microstructure against TCP precipitation $[3,4]$. This study was performed to isolate the effect of these four elements as well as their interaction with each other, since elements like $\mathrm{Ru}$ can influence the partitioning behavior of other alloying elements $[5,6,7]$. 


\section{Experimental method}

Eight $4^{\text {th }}$ generation single crystal Ni-base superalloys with a high concentration of Re and varying contents of $\mathrm{Ru}, \mathrm{Co}, \mathrm{Mo}$ and $\mathrm{W}$ were cast into $<001>$ oriented single crystal bars at the Precision Casting Facility (PCF) in Rolls-Royce plc, Derby, UK. The content of each of the four alloying elements under investigation was chosen to be either low or high in the different alloys. The compositions of the alloys LDSX 1 to 8 are given in Table 1 . All alloys were solution heat treated at $1340^{\circ} \mathrm{C}$ for $10 \mathrm{hrs}$ and LDSX 3 and LDSX 8 for an additional 10 and $5 \mathrm{hrs}$ respectively due to their high content of $\mathrm{W}$ and Mo. LDSX 1 was solution heat treated at $1355^{\circ} \mathrm{C}$ due to its higher $\gamma^{\prime}$ solvus temperature. All alloys were subsequently aged at $1150^{\circ} \mathrm{C}$ for $4 \mathrm{hrs}$ and at $870^{\circ} \mathrm{C}$ for $16 \mathrm{hrs}$ (hereinafter referred to as standard heat treated state).

Table 1: Nominal alloy compositions of the different LDSX alloys in atomic percent (at.\%).

\begin{tabular}{lccccccccccc}
\hline & Ni & Cr & Co & Mo & W & Ta & Al & Ti & Re & Ru & Hf \\
\hline LDSX 1 & bal. & 3.59 & 3.17 & 1.62 & 0.98 & 2.23 & 13.83 & 0.32 & 2.07 & 2.15 & 0.03 \\
LDSX 2 & bal. & 3.63 & $\mathbf{8 . 5 3}$ & $\mathbf{3 . 2 8}$ & 0.99 & 2.26 & 13.98 & 0.33 & 2.09 & 2.18 & 0.04 \\
LDSX 3 & bal. & 3.68 & 3.24 & $\mathbf{3 . 3 2}$ & $\mathbf{1 . 6 6}$ & 2.29 & 14.17 & 0.33 & 2.12 & 2.21 & 0.04 \\
LDSX 4 & bal. & 3.64 & $\mathbf{8 . 5 6}$ & 1.64 & $\mathbf{1 . 6 5}$ & 2.27 & 14.03 & 0.33 & 2.10 & 2.18 & 0.04 \\
LDSX 5 & bal. & 3.61 & $\mathbf{8 . 5 0}$ & 1.63 & 0.99 & 2.25 & 13.93 & 0.33 & 2.09 & $\mathbf{3 . 1 0}$ & 0.04 \\
LDSX 6 & bal. & 3.66 & 3.23 & 1.65 & $\mathbf{1 . 6 6}$ & 2.28 & 14.12 & 0.33 & 2.11 & $\mathbf{3 . 1 4}$ & 0.04 \\
LDSX 7 & bal. & 3.65 & 3.22 & $\mathbf{3 . 3 0}$ & 1.00 & 2.27 & 14.07 & 0.33 & 2.11 & $\mathbf{3 . 1 3}$ & 0.04 \\
LDSX 8 & bal. & 3.70 & $\mathbf{8 . 7 1}$ & $\mathbf{3 . 3 4}$ & $\mathbf{1 . 6 8}$ & 2.31 & 14.27 & 0.34 & 2.14 & $\mathbf{3 . 1 7}$ & 0.04 \\
\hline
\end{tabular}

For TEM investigations $\sim 0.1 \mathrm{~mm}$ thin slices were electropolished with a solution of $10 \%$ perchloric acid in methanol at $\sim-5^{\circ} \mathrm{C}$ and $25 \mathrm{~V}$. The microstructural investigations were performed by using a JEOL 200CX TEM. For the XRD measurements the samples were mechanically ground and polished and analysed with a high resolution Philips/Panalytical PW3050/65 X'Pert PRO HR horizontal diffractometer at room temperature. The X-ray beam was monochromatised with a Ge (220) 4-bounce primary monochromator and the $\{002\}$ reflection recorded with a 3-bounce analyser crystal. $\omega-2 \theta$ scans were performed within a $2 \theta$ range from $50^{\circ}$ to $51.5^{\circ}$ with a step size of $0.02^{\circ}$ and a $\Delta \omega$ range of $6^{\circ}$. The recorded $\{002\}$ reflections were fitted by using three symmetric Pseudo-Voigt, V(a), functions, each being the weighted sum of a Gaussian, G(a), and a Lorentzian, L(a), function (Eq. 1):

$$
\begin{aligned}
& V(a)=I_{0}[(1-n) G(a)+n L(a)] \\
& G(a)=\exp \left[-\ln (2) \cdot\left(\frac{a-a_{0}}{\omega}\right)^{2}\right] \\
& L(a)=\frac{1}{1+\left(\frac{a-a_{0}}{\omega}\right)^{2}}
\end{aligned}
$$

Similar to the work of Diologent et al. [8] on a $4^{\text {th }}$ generation nickel-base superalloy, three PseudoVoigt-functions were required to fit the experimentally measured X-ray peaks. The lattice misfit was derived from the resultant lattice parameters of the $\gamma$ and $\gamma^{\prime}$ phase respectively, which is discussed in more detail in the following section. Further information on the $\omega-2 \theta$ scans and additional investigations on the high temperature lattice misfits of $4^{\text {th }}$ generation Ni-base superalloys will be presented in another work [9]. 


\section{Results and Discussion}

The diffraction profiles of the highly alloyed superalloys (Fig. 1) possess a broader X-ray profile than former nickel-base superalloys $[10,11,12]$. In comparison with less alloyed nickel-base superalloys the alloys LDSX 1 and 6 already show two separate peaks in the as heat treated condition and in LDSX 2, 3, 7 and 8 three peaks may be unambiguously distinguished.

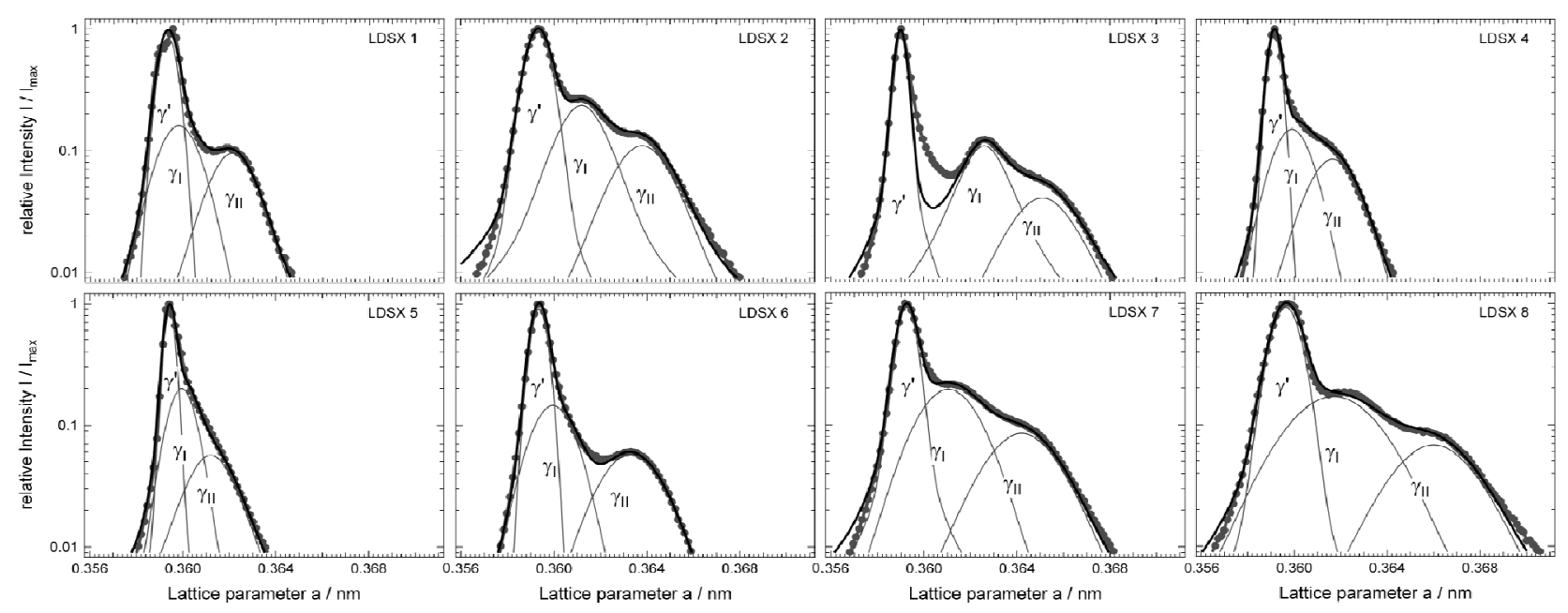

Figure 1: $\{002\}$ reflections and the three sub-peaks $\left(\gamma^{\prime}, \gamma_{\mathrm{II}}\right.$ and $\left.\gamma_{\mathrm{II}}\right)$ of all alloys LDSX 1 to 8 at room temperature. LDSX 3 couldn't be fitted properly with three symmetric Pseudo-Voigt functions, the origins of which will be discussed in a further paper [9].

As pointed out by Diologent et al. in a similar study on one $4^{\text {th }}$ generation nickel-base superalloy [8], this phenomenon can be attributed to two possible sources. First, residual chemical segregation at the dendrite scale and, second, the internal stresses generated by the lattice misfit, which induce a tetragonal lattice distortion of the $\gamma$ phase.

Earlier convergent beam electron diffraction (CBED) and X-ray measurements on Re-containing alloys have shown that the lattice misfit varies strongly within the alloy due to an inhomogeneous Re distribution. This element segregates preferentially to the solid during solidification, resulting in Re-enriched dendrite cores that are not readily homogenised by solution heat treatment $[13,14]$ due to its strong segregation behavior [15] and its low diffusion coefficient [16]. Additionally, $\mathrm{Re}$ partitions to the $\gamma$ matrix phase during precipitation of $\gamma^{\prime}[6,10]$. As a consequence of these effects Re causes a much bigger increase of the $\gamma$ lattice parameter in the dendrite core than in the interdendritic region. This could result in two distinguishable matrix peaks $\gamma_{\mathrm{I}}$ and $\gamma_{\mathrm{II}}$. In addition, the lower concentration of $\operatorname{Re}$ in the $\gamma^{\prime}$ precipitate phase would not be expected to lead to significant variations in the lattice parameter of the $\gamma^{\prime}$ on the dendrite scale. Accordingly, the $\gamma^{\prime}$ phase contributes only one distinct peak to the $\{002\}$ X-ray profile. Consequently a huge difference of the lattice misfit between dendrite core and interdentritic regions exists which leads to different microstructures due to the different coherency stresses (Fig. 2).

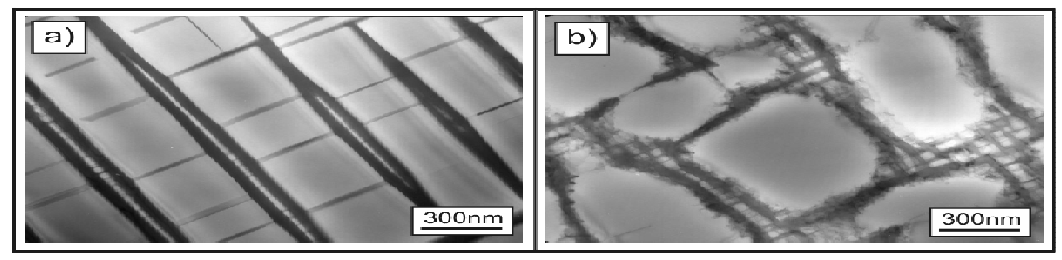

Figure 2: TEM (bright field) images of different regions in LDSX 8. Due to an inhomogeneous element distribution the microstructure varies within the alloy. In some regions (see Fig. b) interfacial dislocations can be found already in the standard heat treated state to reduce the high coherency stresses due to the high lattice misfit. 
Internal stresses arising as a result of high lattice misfit may also account for the observed behaviour. Kuhn et al. pointed out that the matrix channels running perpendicular and parallel to the stress axis [001], respectively, contribute in different ways to the reflection profile due to the tetragonal distortion of $\gamma$ due to coherency stresses [17]. Neutron diffraction measurements on homogeneous Ni-Co-Al-Ti alloys with high lattice misfit [18] and X-ray experiments by Royer et al. [19] have confirmed that high coherency stresses in the matrix channels leads to a tetragonal distortion of the $\gamma$ matrix.

The fact that all alloys investigated in this study possess the same Re content and have been subjected to similar solution heat treatments suggests that a similar residual Re segregation may be expected between the alloys. Additionally, the observation that LDSX 2 and LDSX 4 exhibit very different peaks confirms that the shape of the peaks can't be attributed only to the elemental inhomogeneity. Both alloys differ only in the Mo and W content. LDSX 2 has a high Mo and low $\mathrm{W}$ and LDSX 4 a high $\mathrm{W}$ and low Mo content. Due to the higher diffusion coefficient of Mo in Ni than $\mathrm{W}$ in $\mathrm{Ni}$ [16] and a less pronounced segregation behavior of Mo [15] any residual Mo and W segregation should be less in LDSX 2. However LDSX 2 clearly shows two separate peaks for the $\gamma$ matrix, in contrast to LDSX 4. This observation may, however, be readily explained by the tetragonal distortion of the $\gamma$ matrix phase. W partitions almost equally between $\gamma$ and $\gamma^{\prime}$, whereas Mo partitions predominantly to the $\gamma$ matrix phase $[6,8,10]$. This leads to a much higher negative lattice misfit in LDSX 2 and hence to much higher coherency stresses, which causes a stronger lattice distortion of the $\gamma$ matrix phase and therefore the horizontal and vertical matrix channels generate two separate $\gamma$ matrix peaks $\gamma_{\mathrm{I}}$ and $\gamma_{\mathrm{II}}$. However, the alloys also possess different channel widths including very small $\gamma^{\prime}$ precipitates in some of the $\gamma$ channels, as shown in Fig 2., which might have an additional influence on the shape of (002) reflections. Nevertheless, further studies on fully homogenized samples without any residual segregation, which will be presented elsewhere [9], corroborate the assumption that the tetragonal distortion of the $\gamma$ phase can lead to three peaks. In Fig. 3 the influence of the elements Ru, Co, Mo and W on the lattice parameter $\mathrm{a}_{\gamma^{\prime}}$ of the $\gamma^{\prime}$ phase as well as on $\mathrm{a}_{\gamma \mathrm{I}}$ and $\mathrm{a}_{\gamma \mathrm{rI}}$ of the $\gamma$ phase is shown.

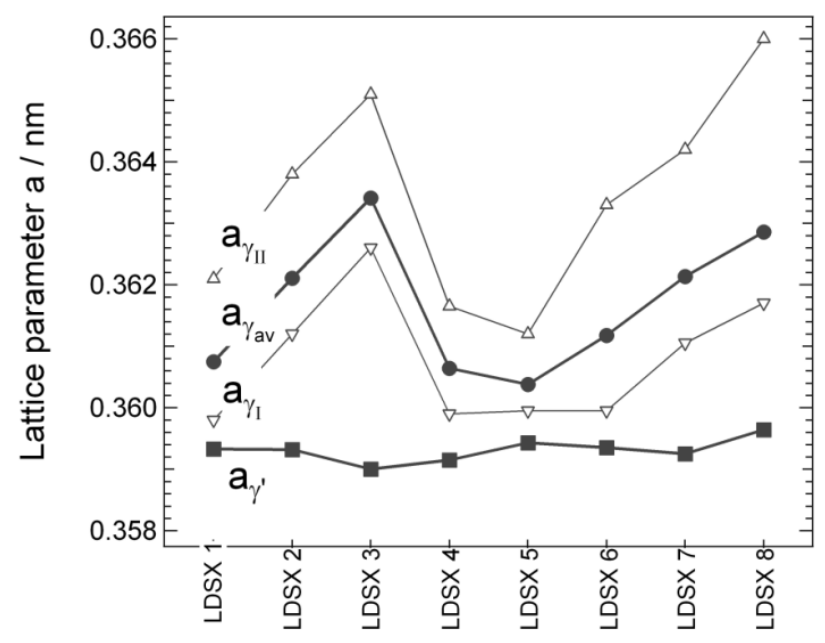

Fig. 3: Lattice parameters of the $\gamma$ and $\gamma^{\prime}$ phase of the different LDSX alloys.

The average lattice parameter $a_{\text {yav }}$ of the $\gamma$ matrix has been calculated by taking an average of the lattice parameters weighted by the area of each matrix sub-peak using:

$$
a_{\gamma a v}=\frac{a_{\gamma I} I_{\gamma I}+a_{\gamma H} I_{\gamma I I}}{l_{\gamma I}+l_{\gamma H}}
$$

where $\mathrm{I}_{\gamma \mathrm{I}}$ and $\mathrm{I}_{\gamma \mathrm{II}}$, respectively are the integrated intensities of the $\gamma_{\mathrm{I}}$ and $\gamma_{\mathrm{II}}$ peaks. The ratio of the sum of the integrated intensities, $I_{\gamma \mathrm{I}}$ and $\mathrm{I}_{\gamma \mathrm{II}}$ to the integrated intensity of the $\gamma^{\prime}$ subpeak, $\mathrm{I}_{\gamma^{\prime}}$, varies between 50:50 and 40:60 which corresponds approximately to the ratio of the $\gamma$ and $\gamma^{\prime}$ volume 
fraction of the alloys. In contrast to the lattice parameter of the $\gamma$ matrix, the lattice parameter of the $\gamma^{\prime}$ precipitate phase is nearly unaffected by the different contents of the alloying elements $\mathrm{Ru}, \mathrm{Co}$, Mo and $\mathrm{W}$. This is to be expected when the effect of these alloying elements on both phases is considered. First, Co has almost no influence on the lattice parameter of both $\gamma$ and $\gamma^{\prime}$ [20]. Second, the Vegard coefficients of Ru, Mo and $\mathrm{W}$ are approximately 2 times smaller for the $\gamma^{\prime}$ phase than the $\gamma$ phase [20] and $\mathrm{Ru}, \mathrm{Co}$ and Mo partition mainly to the $\gamma$ phase [10,6]. Although $\mathrm{W}$ partitions more equally between both phases $[8,10]$ the additional content of $\mathrm{W}$ is small compared to $\mathrm{Ru}$ and Mo. Another factor is a possible influence of $\mathrm{Ru}$ on the partitioning behavior of other alloying elements, like Re, which has been reported in different studies [5, 6, 7]. Despite the stronger tendency of Ru to partition to the $\gamma$ matrix than $\mathrm{W}$ and a higher additional content of Ru in LDSX 7 than W in LDSX 3, alloy LDSX 7 has a smaller $\gamma$ lattice parameter but a bigger $\gamma^{\prime}$ lattice parameter than LDSX 3.

A similar trend can be found between LDSX 5 and LDSX 4. This indicates that Ru leads to a change in the partitioning behavior of other elements, increasing their concentration in the $\gamma^{\prime}$ precipitates and slightly decreasing their concentration in the $\gamma$ matrix. Due to the partitioning behavior of $\mathrm{Mo}$, the relatively strong influence on the lattice parameter of the $\gamma$ matrix and a strong additional increase in the Mo concentration, the high Mo containing alloys LDSX 2, 3, 7 and 8 all possess a high $\gamma$ matrix lattice parameter.

The extremely different lattice parameters of the $\gamma$ matrix phases in the alloys investigated lead accordingly to different lattice misfits. The average lattice misfits have been calculated by the following formula [8] and are plotted in Fig. 4:

$$
\delta_{a v}=\frac{\delta_{\gamma I} I_{Y I}+\delta_{Y I I} I_{Y I I}}{I_{Y T}+I_{Y n}}
$$

where $\delta_{\gamma l}$ and $\delta_{\gamma l l}$ are the lattice misfits between $\gamma^{\prime}$ and $\gamma_{\mathrm{I}}$ and $\gamma^{\prime}$ and $\gamma_{\mathrm{II}}$, respectively.

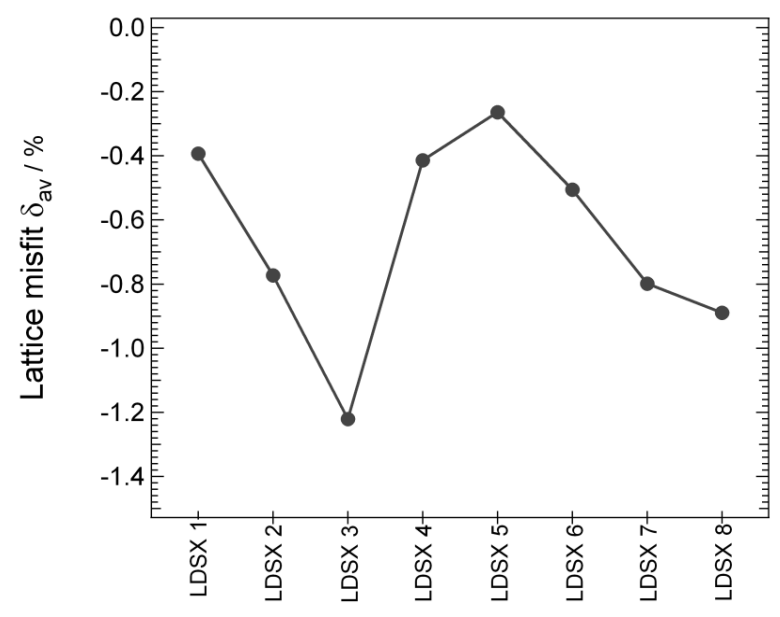

Fig. 4: Average lattice misfit $\delta_{a v}$ of alloys LDSX 1 to 8 .

All experimental alloys exhibit a large negative lattice misfit at room temperature. The largest misfits are seen in the alloys with the highest Mo contents. This is consistent with the loss of coherency observed in some regions of LDSX 2,3,7 and 8 in the as heat treated condition (Fig. 2).

\section{Summary}

The influence of high and low contents of $\mathrm{Ru}, \mathrm{Co}$, Mo and $\mathrm{W}$ on the lattice parameter and lattice misfit in high rhenium containing single crystal nickel-base superalloys have been determined by $\mathrm{X}$-ray diffraction. From this work the following conclusions can be drawn: 
The lattice parameter of the $\gamma$ and $\gamma^{\prime}$ phase is influenced by the content of the elements $\mathrm{Ru}$, Co, Mo and $\mathrm{W}$, their partitioning behavior, their effect on the partitioning behavior of other elements and their Vegard coefficients for $\gamma$ and $\gamma^{\prime}$.

The highly alloyed nickel-base superalloys posses a huge variety in average lattice misfit from $-0.25 \%$ to $-1.22 \%$.

Due to a residual segregation on the dendrite scale and a coherency stress induced tetragonal distortion of the $\gamma$ matrix two distinguishable peaks for the $\gamma$ matrix are observed in the $\{002\}$ $\mathrm{X}$-ray reflections.

\section{Acknowledgements}

The authors gratefully acknowledge Rolls-Royce plc., the Engineering and Physical Sciences Research Council (EPSRC) for provision of material and funding, Mary Vickers at the X-ray facility at University of Cambridge and Lijuan Zhang for the TEM images.

\section{References}

[1] J.X. Zhang, J.C. Wang, H. Harada, Y. Koizumi: Acta. Mat. 53 (2005) p. 4623-4633.

[2] R.A. Hobbs, G.J. Brewster, C.M.F. Rae and S. Tin in: Superalloys 2008, edited by R.C. Reed et al., TMS, Warrendale, PA (2008), p.171-181.

[3] A.C. Yeh and S. Tin: Met. Mat. Trans. A 37A (2006) p. 2621.

[4] W.Z. Wang, T. Jin, J.L. Liu, X.F. Sun, H.R. Guan and Z.Q.Hu: Mat. Sci. Eng. A 479 (2008) p. 148-156.

[5] L.J. Carroll, Q. Feng, J.F. Mansfield and T.M. Pollock: Mat. Sci. Eng. A 457 (2007) p. 292-299

[6] S. Neumeier, F. Pyczak, M. Göken in: Superalloys 2008, edited by R.C. Reed et al., TMS, Warrendale, PA (2008), p. 109-119.

[7] F.Pyczak, S. Neumeier, M. Göken: Mat. Sci. Eng. A. 527 (2010), p. 7939-7943.

[8] F. Diologent, P. Caron, T. D’Almeida, S. Chambreland, A. Jacques and P. Bastie: Int. J. Mat. Res. 97 (2006) 8, p. 1136-1142.

[9] S. Neumeier, C.M.F. Rae, H.J. Stone: to be published.

[10] F. Pyczak, B. Devrient and H. Mughrabi in: Superalloys 2004, edited by K.A. Green et al., TMS, Warrendale, PA (2004), p. 827-836.

[11] B. v.Grossmann, H. Biermann, U. Tetzlaff, F. Pyczak and H. Mughrabi: Scripta Mat. 43 (2000) p. 859-864.

[12] F. Pyczak, S. Neumeier, M. Göken: Mat. Sci. Eng. A 510-511 (2009) p. 295-300.

[13] R. Völkl, U. Glatzel and M. Feller-Kniepmeier: Acta mater. Vol. 46, No. 12 (1998) p. 4395-4404.

[14] U. Brückner, A. Ephishin and T. Link: Acta mater. Vol 45, No. 12 (1997), p. 5223-5231

[15] A. Heckl, R. Rettig, R.F. Singer: Met. Mat. Trans. A 41A (2010) p.202-211

[16] C.L. Fu, R. Reed, A. Janotti and M. Krcmar in: Superalloys 2004, edited by K.A. Green et al., TMS, Warrendale, PA (2004), p. 867-876.

[17] H.-A. Kuhn, H. Biermann, T. Ungar, H. Mughrabi: Acta metall. Mater. 39, 11 (1991) p. 2783-2794.

[18] J.P. Minshull, S. Neumeier, M. Tucker, H.J. Stone: published in this issue

[19] A. Royer and P. Bastie in: Superalloys 1996, edited by by R.D. Kissinger et al., TMS, Warrendale, PA (1996), p.221-228.

[20] R. Reed. The Superalloys. Cambridge, 2005. 